\title{
Computer and Computer Communication Guidance Centre for the Disabled
}

\author{
Dr. P.D. Bhalerao \\ Project Leader, Dept. of Computer Science, Institute of Science, R. Tagore Rd, NAGPUR \\ INDIA 440001.

\begin{abstract}
Computer and Computer Communication Guidance Centre for Disabled is started at the Institute of Science, Nagpur India with the financial assistance from UNCSDHA Vienna and infrastructural facilities from State Govt. of Maharashtra. This is a highly motivated project that focusses on the upliftment of disabled to the status of economic independence through free of charge high tech. education in the field of computers. In fourteen months fifty disabled have been trained and referred for job placement. Other objectives include Computer aided diagnosis of medical and psychological health of disabled, adoptions and attachments for regional languages, training in D.T.P., FAX and EPABX as well development of incidental software.
\end{abstract}

\section{Introduction}

Self relience and respectable status in profession/career for the physical handicapped has been as a noble goal in various civilization and cultures of the world. In the last decade in India and the South-East Asian countries awareness for computer and computer communication systems in on the increase. A wide range of new professions are being thrown open in this field. It is, therefore time that adoptions and training in this field for the handicapped of different categories is worked out. The centre is located at the Govt. Institute of Science, a prime educational institute in central India since last eight decades with higher education in Physics, Electronics, Computer Science, Maths, Chemistry, Botany, Zoology, Statistics and Environment science. Presently the centre is equipped with 486 AT, a LAN with four 286 nodes, 4 XT's, 3 DMP's, Speech System from COVAX U.S.A., Language Card from CDAC for regional languages, DTP facilities and related software. An offer is received from Robotron Australia for lending a braille computer and text reader. We are approaching funding agencies for obtaining the facilities permanently.

\section{Objectives}

A) Vocational evaluation and adjustment of handicapped persons to the growing world of personal and mini computer and computer communication world.

B) Development of computer and computer communication systems with suitable attachments for adoption of disabled persons to take computer training and help them rehabilitate in approprite vocational/selp employed jobs.

C) To set up computer aided diagnosis of the medical and psychological health for the disabled. 
D) To constitute an R\&D Base for incidental hardware \& software development.

E) Adoptations of attachments for regional language so that disabled can record better pick-up of training.

\section{Activities}

The Project is started with the resources provided by United Nations(US $\$ 20,000$ ) and the basic infrastructure and support from the State Government of Maharashtra. This is a highly motivated project that focuses on the upliftment of disabled to the status of economic independence through free high tech education in the field of computers. We have trained fifty disabled persons in computer use during a period of fourteen months. Ten of them have been employed. Some more students are referred for job placement and some are starting their own business with computer related jobs. With the hardwork put in by our dedicated team the project is successful in creating basic infrastructure for training of disabled during the very first year even though its tennure is of three years.

Since disabled students are drawn mainly from the lower middle class and poor families, who cannot affored costly cducation in new technologies like computer, the centre provides free education to all disabled students as a policy matter. A new batch of ten employed disabled has also started from 1st Jan. 1994 to improve their promotional chances in service. The centre is presently training deaf and dumb student to use the speech system for communicating their thoughts. The centre gives four weeks short term computer training free to Maharashtra Government employees deputed from various departments. Senior batch disabled students help in this training. A software has been developed to solve the non linear auto regressive time series (ARTS) which generates various patterns. These patterns ARTS simulated are useful in textile -Industry. Progness is made in the development of software to translate English text into regional languages with limited vocabulary. A Project on OCR is in pipeline in collaborations with IIT, Bombay.

\section{Conslusions}

In order to meet the growing demand of educating more disabled students we need to update and increase the infrastructural facilities at the centre. Similarly there is a requirement to constantly train our staff in keeping pace with the latest technology as well as make new technologies available at our centre for the benefit of the disabled.

\section{Acknowledgements}

Our Acknowledgement are due to United Nations Social Development Division Vieena, Govt. of Neitherlands and State Govt. of Maharashtra for th.ir aid in this project. 